\title{
Vector bundles on the noncommutative torus from cochain quantization
}

\section{Francesco D'Andrea*}

University of Naples Federico II

E-mail: francesco.dandrea@unina.it

\begin{abstract}
The non-commutative torus is commonly described as a cocycle quantization of the group $\left(C^{*}\right.$ )algebra of the abelian group $\mathbb{Z}^{2}$. In the first part of the talk I will explain how, using the WBZ transform of solid state physics, finitely generated projective modules over the noncommutative torus can be interpreted as deformations of vector bundles on elliptic curves by the action of a 2-cocycle, provided that the deformation parameter of the NC-torus and the modular parameter of the elliptic curve satisfy a non-trivial relation.

I will then discuss the relation between (formal) deformations of vector bundles on the torus and cochain twists based on the Lie algebra of the 3-dimensional Heisenberg group.

Based on a joint work with G. Fiore and D. Franco.
\end{abstract}

Frontiers of Fundamental Physics 14 - FFP14,

15-18 July 2014

Aix Marseille University (AMU) Saint-Charles Campus, Marseille

\footnotetext{
*Speaker.
} 